\title{
Routing Metrics for Wireless Mesh Networks: a survey
}

\author{
Liang Zhao, Ahmed Y. Al-Dubai \\ School of Computing, Edinburgh Napier University \\ Edinburgh, EH10 5DT, UK \\ Email: \{l.zhao, a.al-dubai\}@napier.ac.uk
}

\begin{abstract}
Wireless Mesh Network (WMN) is a key emerging technology to construct next generation wireless multi-hop network. It combines the advantages of both mobile ad-hoc network (MANET) and traditional fixed network, attracting significant industrial and academic attentions. In network layer, routing metrics are also important to the performance of communication. In this paper, we review a list of existing routing metrics in WMNs. Based on the findings, we also point out the open issues of routing metrics.
\end{abstract}

Keywords: routing metric, wireless mesh network.

\section{INTRODUCTION}

Wireless Mesh Network (WMN) is becoming a major avenue for the next generation wireless networks. This is due mainly to the revaluation of using basic radio frequency physics to provide a robust, flexible, standard-based architecture. Usually, this architecture offers instant, highly flexible, and low-cost mobile broadband communications to different communities through the readily attainable multihop connection. In WMNs, signals are routed optimally and nodes can automatically join and leave the network at any time. Furthermore, networks can be established instantly virtually anywhere, even in places with no fixed infrastructure. Instead of using optical fibre cable, wireless radios are applied in WMNs. They have been already deployed to build wireless broadband network in some newly developing areas worldwide [1] and isolated islands [2]. A WMN combines the characteristics of both fixed network and MANET. The communication inside a WMN is similar to MANET, client nodes are self-configured and self-organized where the routes are selected by using certain routing algorithm and each client node has to relay other's packets. For accessing the backbone internet, the packets are forwarded through internet gateway to the fixed network by fixed cable links.

In the implementation of routing protocols, routing metrics are assigned to different paths. It calculates the cost of each path in order to select or predict the best path. This path is then stored in the routing table for future use. Routing metrics are integrated in routing protocols to improve communication quality in term of bandwidth, error rate, latency, reliability, and cost. In addition, the improvement of one aspect normally results in all other aspects. For example, a good communication cost routing metric also performs well in reducing delay etc. As mentioned in [3], new routing metrics are required to examine and improve the performance of WMNs in dealing with more constraints. Because channel bandwidth is limited in wireless communication, the design of routing metrics is important.

In this paper, we first review the performance of a list of existing routing metrics, Hop-count [11] [12] [13], ETX [9], SPP [6], Load-count [7] [8], and WCETT-LB [14] and so forth. We also describe the possible open issues remain in the current design of routing metrics in WMNs.

\section{NETWORK MODEL}

WMNs are a particular type of Mobile Ad-hoc Networks (MANET). A WMN consists of mesh clients, mesh routers and gateways where mesh routers and mesh clients are designed to increase the coverage of WMNs by only using wireless radio while a gateway use both wireless radios and fibre optic cable. Mesh clients connect to gateways through mesh routers, while mesh routers connect to a backbone network via gateways and gateways relay the message from internet to the mesh clients. Hence, there are three layers in a typical wireless mesh network: Internet Gateway Layer (IGW), Mesh Router Layer (MR) and Mesh Client Layer (MC) [14] as depicted in Figure 1. To simplify the description of the routing algorithm, we combine the MR layer and MC layer in this paper as both MR and MC are routing devices that use wireless radio and are connected to the backbone of the internet via gateways.

There are two major differences between WMN and MANET which are gateway and mobility. Compared to MANET, most of the traffic is expected to flow between the mesh clients and the backbone network through gateways. Furthermore, in MANET, all the nodes are assumed as mobile nodes moving in the network. In contrast with MANET most of devices in WMNs are stationary or with limited mobility, where only a small portion of devices such as mobile phones, are moving in the network. The characteristics of WMN are not considered in the existing routing protocols such as AODV [11], DSR [12] and DSDV [13], and most of the existing metrics such as HOP COUNT [11] and ETX [9][10] etc. In fact, the previous protocols and metrics are designed with particular attention paid to the mobility of the nodes, i.e., supposing most of nodes are highly mobile. Therefore, both new routing protocol and metrics are required to be designed for WMNs, the old routing protocol and metrics also have to be re-engineered to satisfy requirements of WMNs. 


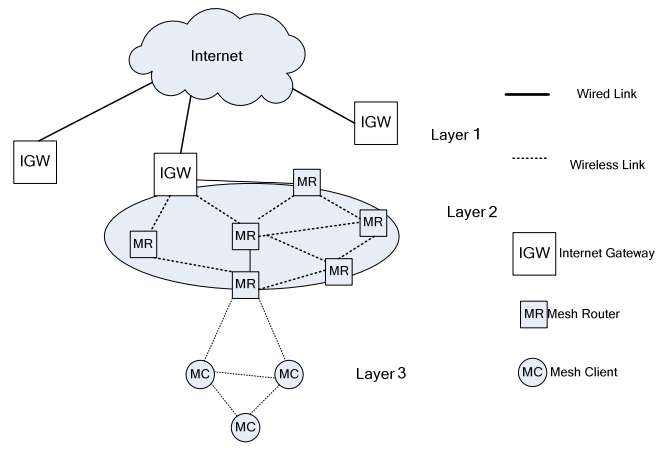

Figure 1: The network structure of a typical WMN

\section{EXISTING Routing Metrics}

Because channel bandwidth is limited in wireless communication, the design of routing metrics is important. Existing routing metrics can be classified into following types: distance, latency, traffic load, error rate, multiplechannel, channel usage and compositive metric. The following sub-sections show typical examples of each type.

\section{(a). Distance routing metric}

Hop-count is widely used in existing protocols such as AODV [11], DSR [12], and DSDV [13]. A routing protocol with the Hop-count metric considers the number of hops between source and destination. Hence, it finds the path with the minimum distance. However, it does not consider other issues such as link quality, transmission rates. Since minimizing the number of hops is not usually the performance goal in WMNs, Hop-count may result in poor performance.

\section{(b). Latency routing metrics}

Per-hop Round Trip Time (RTT) [4] is designed for Multi-Radio Unification protocol. It measures the round trip delay of unicast probes between neighbours. In this metric, each node sends out a probe packet with timestamp to all neighbours. When receiving the probe packet, each neighbour may respond an acknowledgement. As sender receives the acknowledgement, it calculates the round trip time between sending probe and receiving acknowledgement. It avoids busy channel and link loss by the path selection. However, queue delay exists due to the contention among nodes for low RTT link. RTT also generates high overhead and self interference.

Per-hop Packet Pair Delay (PktPair or PP) [9] is an improved version of round-trip time (RTT) by considering queue delay and transmission rates. In PktPair, a node sends out two probe packets to each neighbour every 2 seconds. In addition, the first probe packet is small (137 bits) and the second is large (1137 bits). This tests the sensitivity of link bandwidth for packets in different sizes. Once receiving probes, each neighbour calculates the delay difference of these two packets and reports the delay result to the sender. Sender keeps the delay result of each of its neighbours for future routing. Although PktPair eliminates the problem of queue delay, it still suffers the self interference and high overhead.

(c). Traffic load routing metric

Load-count [7] [8] is a load balancing metric for wireless networks

$$
\text { Load-count }=\sum_{i=1}^{n} \text { Load }_{i}
$$

where $\operatorname{Load}_{i}$ is the traffic load on a node $i$ which is normally captured by using IFQ length. The IFQ (Network Interface Queue) is a drop-tail buffer at the MAC layer of 802.11 radios, which contains outbound frames to be transmitted by the physical layer where the size of IFQ is calculated as the number of remaining packets in the buffer.

Neighbourhood Load Balancing (NLR) [17] is the average load of each neighbourhood is measured with aim to bypass the busy neighbourhood instead of only bypassing the busy node with Load-count. Moreover, in a heavy loaded neighbourhood, extra traffic on one node influences communication of all nodes within its interference range. The transmission of packets in these nodes can be deferred, or dropped. Thus, there is a side effect caused by allowing a packet goes into heavy loaded neighbourhood. To solve the above problem, NLR is developed to check the summation value of the neighbourhood load over a path which is:

$$
\begin{aligned}
& N L R=\sum_{i=1}^{k} \frac{\operatorname{Load}_{i}^{n}}{b_{i}^{n}} \\
& n=\frac{\operatorname{tr}}{d_{\text {avg }}}
\end{aligned}
$$

where $n$ is the interference radius of neighbourhood in hop number; tr denotes the transmission range (here the transmission range is assumed as same as the interference range), and $d_{\text {avg }}$ is the average distance between two onehop nodes. $\operatorname{Load}_{i}^{n}$ denotes the average load of a neighbourhood of node_ $i$ with radius $n$ hops and $b_{i}^{n}$ is the average transmission rate of this neighbourhood. The neighbourhood size measures average transmission range of the zone with all nodes interference by the centre node. Hence, unlike existing routing metrics, NLR considers three aspects in the selection of the best path, which are IFQ length of each node, neighbourhood interference, and transmission bandwidth.

\section{(d). Error rate routing metric}

Expected Transmission Count (ETX) is a metric to estimate the expected number of MAC layer transmissions for the wireless links and measure the packet loss rate which is proposed by De Couto et al. [9] [10]. A node sends out probe packets to all its neighbour nodes every second. When a neighbour node receives probes, it increments the amount of received packets and calculates the loss rate of packet every 10 seconds. The weight of a route is the sum of the ETX of all links along the path. The possibility of successful packet transmission from source $a$ to destination $b$ in a wireless link is: $p=\left(1-p_{f}\right) \times\left(1-p_{r}\right)$

Then ETX can be achieve as 


$$
E T X=\sum_{k=1}^{\infty} k p^{k}(1-p)^{k-1}=\frac{1}{1-p}
$$

where $p_{f}$ is the probability of successful forwarded packets and $p_{r}$ denotes the probability of successful received packets. The advantages of ETX are the reduced probing overhead and non self-interference as the delay is not measured. However, ETX cannot measure the cause of data size in the delivery ratio and it doest not consider the transmission rate. Furthermore, unicast probing of ETX is not accurate as differences between broadcast and unicast.

Success Probability Product (SPP) [6] is a Routing Metric to enhance the throughput in Wireless Mesh Networks. The authors in [5] modify an energy-efficient routing metric and produce $S P P$ to provide the probability for the destination node to receive a packet over a link.

$$
S P P=\prod_{i=1}^{n} d_{f_{i}}
$$

where $d_{f_{i}}=1-p_{\text {err }}, d_{f_{i}}$ has been already mentioned above in ETX as the probability of transmission and $p_{\text {err }}$ is the error rate of a link $i$. Compared to other metrics, a good path has to have a high value of $S P P$ and a bad path has a low value of SPP. SPP has the same problem as ETX as it does not consider packet size and link bandwidth.

Airtime link metric [30] determines the routing prospect of each paired nodes. It is defined as the amount of channel resources consumed by transmitting the frame over a particular link. The Airtime Link metric of a link is

$$
C_{a}=\left[O+\frac{B_{t}}{r}\right] \frac{1}{1-e_{f}}
$$

where $O$ is the variation depends on the channel access overhead in the physical layer, including frame headers, training sequences, access protocol frames, etc. $B_{t}$ is size of test frame. $e_{f}$ denotes the frame error rate. It is the possibility of transmission error on $B_{t}$ data size packet at the bit rate $r$. The main disadvantage of this metric is it generates high probing overhead.

\section{(e). Multi-channel routing metric}

Weighted Cumulative ETT (WCETT) is also proposed by Draves et al [15] and it considers the multi-radio nature of the WMNs in two components: the total transmission time along all hops in the WMN and the channel diversity in the path. The WCETT of a path $p$ is

$$
\operatorname{WCETT}(r)=(1-p) E T T_{l}+p \max _{1 \leq j \leq k} X_{j}
$$

where $p$ is a parameter, $0 \leq p \leq 1$. And path $r$ uses $X_{j}$ number of times of channel $j$. Therefore, $p \max _{1 \leq j \leq k} X_{j}$ denotes the maximum number of times that the same channel $j$ is used along a path. Although it captures the intra-flow interference of a path with measuring the channel assignment time, it does not consider the inter-flow interference. Thus, traffic flows may be routed to the dense area by WCETT. One more important problem of the WCETT is that it is not isotonic which generates a forwarding loop while chosen a path.

\section{(f). Channel usage metric}

Interference-Aware Routing Metric (IAR) [16] detects the channel busy level by capturing the MAC layer information. IAR of a link is

$$
\begin{aligned}
& \operatorname{IAR}(l)=\frac{1}{1-a_{u b}} \times \frac{S}{B} \\
& a_{u b}=\frac{T_{\text {Wait }}+T_{\text {Collision }}+T_{\text {Backoff }}}{T_{\text {Wait }}+T_{\text {Collision }}+T_{\text {Backoff }}+T_{\text {Success }}}
\end{aligned}
$$

where $T_{\text {Wait }}, T_{\text {Collision }}, T_{\text {Backoff }}, T_{\text {Success }}$ are the time spent in Wait, Collision, Backoff and Success states during a packet transmission. The time durations are captured in the MAC layer. $a_{u b}$ is the percentage of time spent in the Wait, Collision and Backoff States compared to the time of completing a transmission. Therefore, smaller IAR presents a path with low traffic.

\section{(g). Compositive metric}

Weighted Cumulative ETT with Load Balancing (WCETT-LB) is a metric proposed by Ma et al. [14] which is an improved version of WCETT. It considers the load balancing in the metric by involving the congestion level which is achieved by calculating the average queue length on each node. However, WCETT-LB has the same problem as WCETT does.

Based on the protocol layers of each metric working on, existing routing metrics can be classified into following three types: single performance parameter metric, singleprotocol-layer metric for multiple performance parameters, and multi-protocol-layer metric for multiple performance parameters. In this context, Hop-count and Load-count are network layer routing metrics. They either capture the number of hops or the traffic load along the paths. Hence, they are single performance parameter metric. IAR is a multi-protocol-layer routing metric for multiple performance parameters. It considers both link layer and network layer to capture MAC handshake time, bandwidth and packet size, respectively. Besides above three routing metrics, all other routing metrics mentioned in this chapter are single-protocol-layer metrics for multiple performance parameters.

To further understand the different characteristics, routing metrics can be also divided into probe-exchange based metric and self-detection metric. Probe-exchange based metrics normally detects the routing status by sending probes in cluster, group or overall network. It normally initiates high overhead. On the contrary, self-detection metrics reduce exchange overhead by only measuring the local routing status. Besides Hop-count, Load-count and IAR, all the routing metrics in this chapter are probeexchange based metrics.

\begin{tabular}{|l|l|l|l|l|}
\hline $\begin{array}{l}\text { Routing } \\
\text { Metrics }\end{array}$ & Layer & $\begin{array}{c}\text { Communication } \\
\text { quality parameters }\end{array}$ & $\begin{array}{l}\text { Power } \\
\text { management }\end{array}$ & QoS \\
\hline $\begin{array}{l}\text { Hop- } \\
\text { count }\end{array}$ & Network & Number of hops & No & No \\
\hline RTT & Network & $\begin{array}{l}\text { Packet loss, delay, } \\
\text { contention }\end{array}$ & No & No \\
\hline
\end{tabular}




\begin{tabular}{|l|l|l|l|l|}
\hline PktPair & Network & $\begin{array}{l}\text { Packet loss, delay, } \\
\text { contention }\end{array}$ & No & No \\
\hline $\begin{array}{l}\text { Load- } \\
\text { count }\end{array}$ & Network & Traffic load & No & No \\
\hline ETX & Network & $\begin{array}{l}\text { Packet loss, } \\
\text { retransmission, } \\
\text { contention }\end{array}$ & No & No \\
\hline SPP & Network & Same as ETX & Yes & No \\
\hline WCETT & Network & $\begin{array}{l}\text { Same as ETX, } \\
\text { plus bandwidth } \\
\text { and packet size }\end{array}$ & No & No \\
\hline IAR & Network, & $\begin{array}{l}\text { MAC handshake, } \\
\text { time, bandwidth } \\
\text { and packet size }\end{array}$ & Yes & No \\
\hline Airtime & Link & $\begin{array}{l}\text { Resource } \\
\text { consumed by a } \\
\text { packet on a link }\end{array}$ & Yes & No \\
\hline $\begin{array}{l}\text { WCETT- } \\
\text { LB }\end{array}$ & Network & $\begin{array}{l}\text { Same as WCETT, } \\
\text { plus traffic load, } \\
\text { bandwidth }\end{array}$ & No & No \\
\hline
\end{tabular}

Table 1: A comparison of different routing metrics for WMNs

\section{OPEN ISSUES}

According to Table 1, there are still several remaining issues in the design of routing metrics for WMNs.

- First of all, many existing routing metrics still work ad-hocly. Consequently, they may only perform well for a certain type of WMN such as Client WMN.

- $\quad$ Second of all, some routing metrics measure routing status inaccurately. For example, ETX abuses the broadcast nature of wireless communication. It uses unicast to measure the error rate of transmission.

- Third of all, probe-exchange based metrics may cause large overhead. It performs especially badly in large scale networks.

- Fourth of all, limited network parameters are considered. Critical parameters such as QoS for diverse applications are not captured in existing routing metrics.

Therefore, it is necessary to design new routing metrics to better optimize the routing protocol so as to achieve better performance.

\section{CONCLUSION AND FUTURE WORK}

Due to the increasing demand for efficient communications, routing metrics should be improved to better work in WMNs. In this paper, we review existing routing metrics for WMNs. In addition, we also describe the remaining issues on the basis of review. In our future work, we will focus on design efficient routing metrics in WMNs.

\section{REFERENCES}

[1]. The Wireless Africa Home Page, 28 Auguest 2008. [Online]. Available:http://wirelessafrica.meraka.org.za/wiki/index.php/Wireless_Afr ica_Home_Page. [Accessed: Sept. 10, 2009].

[2]. N. Lomas, "Greek island gets wireless mesh network", silicon.com, 24 July $2008 . \quad$ [Online]. Available: http://networks.silicon.com/mobile/0,39024665,39263795,00.htm.

[Accessed: Sept. 12, 2009].
[3]. I. Akyildiz and X. Wang, Wireless Mesh Networks. New York: Wiley, 2009.

[4]. Atul Adya, Paramvir Bahl, Jitendra Padhye, Alec Wolman, and Lidong Zhou, "A Multi-Radio Unification Protocol for IEEE 802.11 Wireless Networks," in Proceedings of the First International Conference on Broadband Networks (BROADNETS’04), July 2003, pp. 344 - 354.

[5]. S. Banerjee and A. Misra, "Minimum energy paths for reliable communication in multi-hop wireless networks," in Proceedings of the 6th ACM international symposium on Mobile ad hoc networking and computing, 2002.

[6]. S. Roy, D. Koutsonikolas, S. Das, and Y. C. Hu, "High-throughput multicast routing metrics in wireless mesh networks," in Proceedings of IEEE International Conference on Distributed Computing Systems, 2006.

[7]. D. Nandiraju, L. Santhanam, N. Nandiraju and D. P. Agrawal, "Achieving Load Balancing in Wireless Mesh Networks Through Multiple Gateways," in Proceedings of 2006 IEEE International Conference on Mobile Ad-hoc and Sensor Systems (MASS), pp. 807-812, 2006.

[8]. A. Pirzada, R. Wishart, M. Portmann, J. Indulska, "ALARM: An Adaptive Load-Aware Routing Metric for Hybrid Wireless Mesh Networks," in Proceedings of the 32nd Australasian Computer Science Conference, pp. 25-34, 2009.

[9]. S. Keshav, "A Control-Theoretic Approach to Flow Control," in Proceedings of the conference on Communications architecture \& protocols, pp. 3-15, 1993.

[10]. D. De Couto, D. Aguayo, J. Bicket, and R. Morris, "A HighThroughput Path Metric for Multi-Hop Wireless Routing," in Proceedings of the 9th annual international conference on Mobile computing and networking, pp. 134 - 146, 2003.

[11]. I. D. Chakeres and E. M. Belding-Royer, "AODV Routing Protocol Implementation Design," in Proceedings of the International Workshop on Wireless Ad Hoc Networking (WWAN), Tokyo, Japan, March 2004.

[12]. D. B. Johnson and D. A. maltz, "Dynamic Source Routing in Ad Hoc Wireless Networks,” Mobile Computing, Vol. 353, pp. 153-181, 1996.

[13]. C. Perkins and Bhagwat, "Highly Dynamic Destination-Sequence DistanceVector, Routing (DSDV) for Mobile Computers," in ACM SIGCOMM Computer Communication Review, pp. 234-244, October 1994.

[14]. L. Ma and M. Denko, "A Routing Metric for Load-Balancing in Wireless Mesh Networks," in Proceedings of 21st International Conference on Advanced Information Networking and Applications Workshops, Vol. 2, pp. $409-414,2007$.

[15]. R. Draves, J. Padhye, and B. Zill, "Routing in Multi-Radio, MultiHop Wireless Mesh Networks," in Proceedings of the 10th annual international conference on Mobile computing and networking, pp. 114 128, 2004.

[16]. S. Waharte, B. Ishibashi, R. Boutaba, and D. Meddour, "Interference-aware routing metric for improved load balancing in wireless mesh networks," in Proceedings of 2008 IEEE International Conference on Communications, pp. 2979-2983, 2008.

[17]. L. Zhao, A. Al-Dubai and I. Romdhani. "Novel QoS-Aware Gateway Centralized Multi-hop Routing for Wireless Mesh Networks", 10th IEEE CIT, Bradford, UK, 2010. 